\title{
Magnetically Controlled Photovoltaic Diode Structure
}

V. K. Dugaev ${ }^{1,2}$, Yu. Vygranenko ${ }^{3}$, M. Vieira ${ }^{1}$, V. I. Litvinov ${ }^{4}$, J. Barnaś ${ }^{5}$

${ }^{1}$ Department of Electronics and Communications, Instituto Superior de Engenharia de Lisboa, Rua Conselheiro Emidio Navarro, 1949-014 Lisbon, Portugal

${ }^{2}$ Institute for Materials Science Problems, Vilde 5, 58001 Chernovtsy, Ukraine

${ }^{3}$ Department of Electrical and Computer Engineering, University of Waterloo, Waterloo, Ontario N2L 3G1, Canada

${ }^{4}$ WaveBand Corporation, Suite 1105, 375 Van Ness Ave., Torrance, CA 90501

${ }^{5}$ Department of Physics, Adam Mickiewicz University, ul. Umultowska 85, 61-614 Poznań, and Institute of Molecular Physics, Polish Academy of Sciences, ul. M. Smoluchowskiego 17, 60-179 Poznań, Poland

\begin{abstract}
We propose a new integrated device for spintronics application, which is based on a hybrid metal-semiconductor structure. The device consists of a Si-based $p-i-n$ photodetector sandwiched between two layers of a ferromagnetic metal ( $3 d$ ferromagnet or half-metallic compound). The photocurrent flowing in such a system is shown to depend on its magnetic configuration. This, in turn, allows controlling the device performance by an externally applied magnetic field. We have estimated magnitude of the effect and also determined the role of relevant material parameters.
\end{abstract}

\section{INTRODUCTION}

Recently, many efforts have been undertaken to integrate semiconductors and magnetic metals into magnetoelectronic devices [1]. The main idea is to make use of spin-polarized electrons injected from a magnetic metal into a nonmagnetic semiconductor. If realized, this would enable to design new devices, like for instance magnetically controlled transistors, or others. Unfortunately, the efficiency of spin injection across the junction between a magnetic metal and a nonmagnetic semiconductor turns out to be small because of a large difference in electrical resistivity [2]. From this point of view, the use of ferromagnetic semiconductors instead of metals might resolve the problem. However, the best known ferromagnetic semiconductor, GaMnAs, has critical temperature about $110 \mathrm{~K}$ [3], which is too small for most applications. Therefore, hybrid structures like metal-semiconductor junctions are of great interest. It was shown that the metal-semiconductor incompatibility can be overcome by introducing tunneling barriers at the interface, which can effectively decrease the spin relaxation $[4,5]$.

In this paper we propose a new magnetically controlled photodetector, that consists of the semiconductor $p-i-n$ structure and two thin films of a magnetic metal. The device does not suffer from the metal-semiconductor incompatibility. Magnetic sensitivity of the photoresponse current is of the same physical origin as the giant magnetoresistance (GMR) effect in metallic magnetic multilayers [6-9]. Therefore, this magnetic sensitivity will be referred to in the following as the GMR effect, too. Our estimations and theoretical calculations show that the GMR effect under illumination is much larger than that in equilibrium conditions. The enhancement of GMR is attributed to the illumination induced spin-polarized electron-hole pairs. 
It should be noted that a close idea of using spin-polarized $p$ - $n$ junctions and solar cells has been proposed recently [10]. This structure can be realized in all semiconductor devices for the generation of spin currents.

\section{THE MODEL DEVICE}

The proposed device is the $p-i-n$ structure sandwiched between two magnetic layers, as shown schematically in Fig. 1. The $p-i-n$ structure operates as a usual photodetector; the photocurrent flows when the biased structure is illuminated. The magnetic metallic layers should be thin enough to transmit the light. The key point is that the average relaxation time of spin-up and spin-down electrons and holes in the semiconductor structure depends on the relative orientation of magnetic moments of the outermost metallic layers. The origin of this dependence is the sensitivity of electron scattering from impurities and defects at the interfaces to the spin orientation. Such a dependence is known to be small and only magnetic coating can make it large enough to cause observable effects.

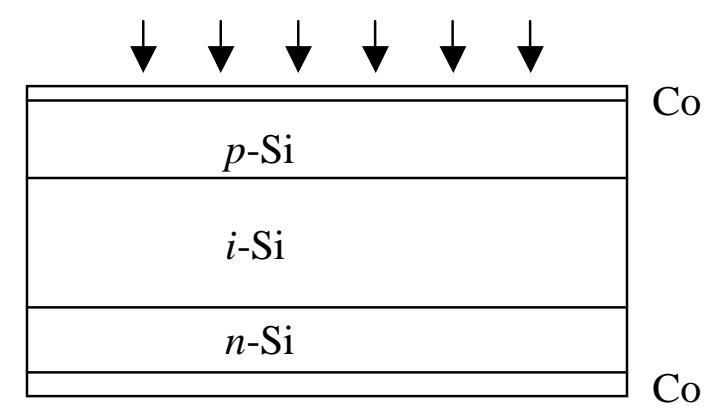

Figure 1. Schematic picture of the proposed structure.

To have a significant contribution due to scattering from interface defects, the thickness $L$ of the semiconductor structure should not be too large. More specifically, it should not be much larger than the mean free path $l$ of electrons and holes in the semiconductor. On the other hand, to make carriers sensitive to both interfaces, $L$ should be of the order of $l$. Thus, we assume $L=\beta l$, where the factor $\beta=3 \div 5$. It is worth noting that for smaller thickness $L$ we can get the dependence of the scattering relaxation time on the magnetization direction in a single magnetic layer, which may also be useful for some applications.

Here, we discuss the $p-i-n$ Si-based implementation of the device. Basically, it is possible to employ a Schottky-barrier structure and other semiconductor materials like GaAs or GaN. In the $p-i$ - $n$ structure the quality of the metal-semiconductor contacts depends on the density of donors and acceptors near the interface. As electrons and holes are generated in the relatively thick undoped $i$-Si region, we have pretty large mean free path (which at $300 \mathrm{~K}$ is limited by phonons). Under illumination the motion of electrons and holes is diffusive. When they reach the region of relatively large electric field, the probability to be scattered back is still very high, even though the probability to move in the direction imposed by the field is larger. The reason of this behavior is large thermal velocity (more than $10^{7} \mathrm{~cm} / \mathrm{s}$ at $300 \mathrm{~K}$ ), which is much larger than the drift one. Thus, due to the diffusive motion, the electrons and holes are sensitive to the scattering from impurities and defects at both interfaces. 
A crucial parameter for the expected magnetic sensitivity of the photocurrent is the spin relaxation time of charge carriers. It should be significantly longer than the corresponding momentum relaxation time. We can expect long spin relaxation time for electrons in the lowdefect $\mathrm{Si}$. However, the spin flip processes at the interfaces remain and may be dominant. Fortunately, in our device the spin-polarized carriers are generated inside the base region, so the interface-induced spin-flip processes are not so critically important as they are when non-equilibrium carriers are injected across the interface. Obviously, a thin tunneling barrier, like a Schottky barrier, could substantially increase the spin relaxation time. As indicated in Fig.1, we propose Co as the magnetic material, for which the Schottky barrier of about $0.8 \mathrm{eV}$ is expected at the interface. It should be noted that since the magnitude of the barrier depends on the bias voltage, the magnetic sensitivity of the photocurrent is expected to be bias dependent, too.

\section{SPIN-DEPENDENT SCATTERING FROM DEFECTS AT INTERFACES}

The spin-dependent average momentum relaxation time in the semiconductor structure determines the sensitivity of the photocurrent to the magnetization orientation. Therefore, we calculated the average momentum relaxation time using the model of charge carrier scattering from defects at the interfaces. The transmission of electrons from a semiconductor to a metal can be calculated as a tunneling rate through a thin Schottky barrier. Due to the large spin splitting in the bulk of a magnetic metal, the transmission and reflection amplitudes depend substantially on the spin orientation of an electron in the semiconductor structure. We calculated the scattering of electrons from a nonmagnetic point defect or impurity located at the interface using the scattering states. The spin-dependent scattering from the interface contributes to the relaxation time, which is given by

$$
\frac{1}{\tau_{k \uparrow, \downarrow}} \approx \frac{2 N_{i} V_{0}^{2} v_{0}}{\hbar L}\left(1+R_{k \uparrow, \downarrow}\right)^{2} \int_{0}^{k_{\max }} d k^{\prime}\left(1+R_{k^{\prime} \uparrow, \downarrow}\right)
$$

where $N_{i}$ is the two-dimensional density of impurities (defects) at the interface, $v_{0}$ is the twodimensional electron density of states, $V_{0}$ is the Fourier transform of the impurity potential, $k$ is the component of momentum perpendicular to the interface, $R_{k \uparrow, \downarrow}$ are the spin-up and down reflection amplitudes from the barrier, and $k_{\max }$ is approximately determined by the thermal electron momentum $k_{\max }=\left(2 m k_{B} T\right)^{1 / 2} / \hbar$.

We have calculated the relaxation time in the structure of thickness $L$, averaged over the distance from the interfaces. A more rigorous approach should take into account the position dependence of the relaxation time, with a characteristic length of the order of $l$. However, as we restrict our consideration to structures of not too large thickness $L$, the approximation we used gives almost the same magnitude of the relaxation time. Equation (1) demonstrates the dependence of the average relaxation time on the spin-dependent reflection from the barrier. With proper choice of the material parameters, the difference between spin-up and spin-down relaxation times can be estimated as large as $10 \%$. 


\section{CALCULATION OF THE GMR EFFECT ON THE PHOTORESPONSE}

To estimate the magnitude of the GMR effect in the photoresponse current, we calculated the effect by taking into account the dependence of the average relaxation time on the relative orientation of magnetizations in the magnetic layers. The semiclassical approximation we used is justified for $k a \gg>1$, where $a$ is the characteristic range of the effective potential. This condition is not valid only near the tunneling barrier.

Under the condition of unpolarized light illumination, the drift components of the photocurrent with parallel $j^{F}$ and antiparallel $j^{A F}$ orientations of magnetization vectors in the magnetic layers determine the GMR effect, which can be characterized by the ratio

$$
\frac{j^{F}-j^{A F}}{j^{A F}} \approx \frac{1}{2} \cdot \frac{1+\left(1+2 \gamma_{a e}\right)^{-1}-2\left(1-\gamma_{a e}\right)^{-1}+g\left[1+\left(1+2 \gamma_{s h}\right)^{-1}-2\left(1+\lambda_{s h}\right)^{-1}\right\rfloor}{\left(1+\gamma_{a e}\right)^{-1}+g\left(1+\gamma_{s h}\right)^{-1}},
$$

where $\gamma_{a e}=\tau_{0 e} / \tau_{a e}, \gamma_{s h}=\tau_{0 h} / \tau_{s h}, \tau$ are the relaxation times of electrons $(e)$ and holes $(h)$ without influence of magnetic layers (labeled with 0 ), and these with magnetization in the layer parallel $(s)$ or antiparallel to the spin $(a)$, respectively. The factor $g$ is given as $g=m_{e} \tau_{0 h} /\left(m_{h} \tau_{0 e}\right)$.

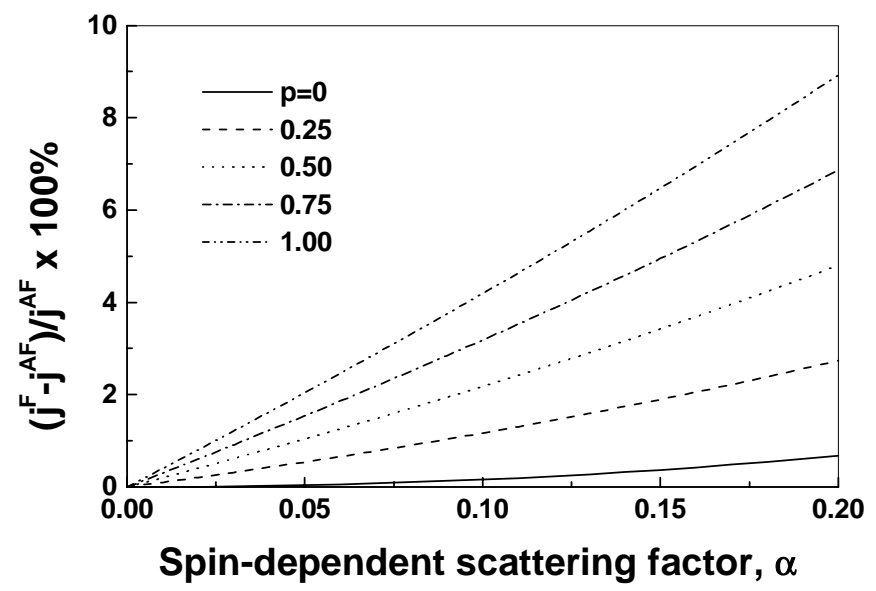

Figure 2. The GMR effect as a function of spin-dependent scattering factor $\alpha$, calculated for different degrees of the light polarization $p$.

Using the material parameters typical of $\mathrm{Si}$, we calculated the sensitivity of the photocurrent to the average spin-dependent scattering factor $\alpha$, which is defined as the coefficient in the spindependent part of the average relaxation time. Results of these calculations are illustrated in Fig. 2, which explicitly shows the importance of polarization of the incident light. For the 100\% polarized light, $p=1$, the effect is one order of magnitude larger than for the unpolarized light. This demonstrates that the photo-GMR effect with polarized light is the first order effect in the effective difference of relaxation times of spin up and down electrons, whereas the usual GMR is known to be the second order effect. 


\section{MODELING}

We simulated the structure with $10 \mathrm{~nm}$ of $p$-Si, $100 \mathrm{~nm}$ of $i$-Si and $10 \mathrm{~nm} n$-Si in contact with Co layers on both sides. We used the standard set of macroscopic equations [11] for the density of electrons and holes, and the electrostatic potential. The calculated potential profile is presented in Fig. 3 for several concentrations of donors and acceptors in $p$ - and $n$-regions and for the temperature $T=300 \mathrm{~K}$.

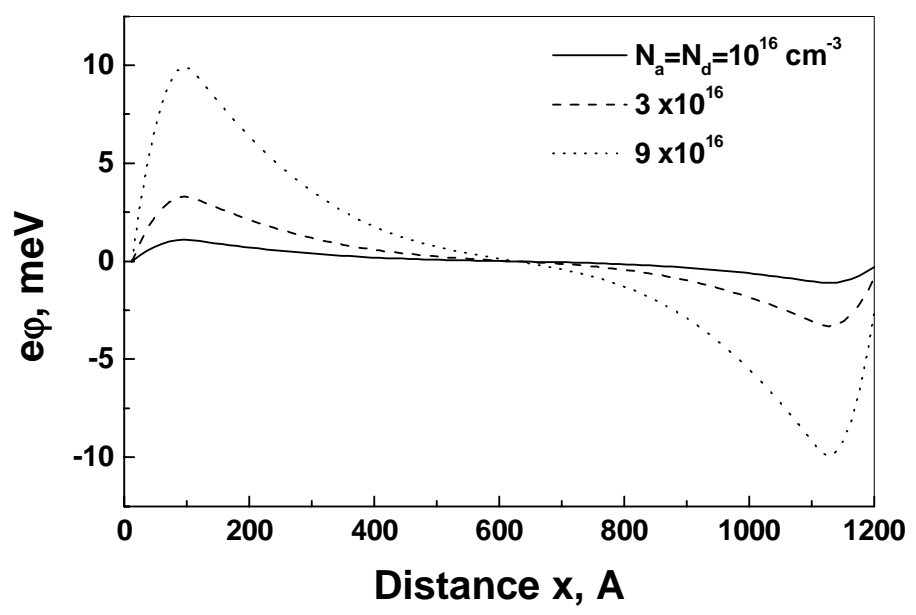

Figure 3. Calculated potential profile in the $p-i-n$ structure.

It should be noted that there is no spin splitting of the conduction and valence bands because of fast spin relaxation of carriers injected through the contact between the magnetic metal and the semiconductor. However, the vanishing efficiency of spin injection from the metal does not affect substantially the work of the proposed device.

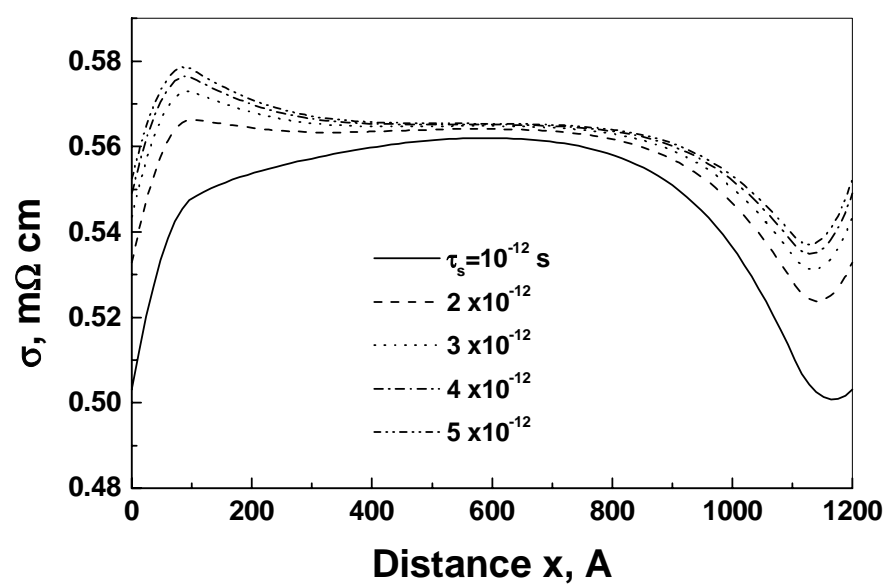

Figure 4. Calculated profile of local conductivity at different scattering rates from the interfaces. 
The profile of local conductivity is presented in Fig. 4. The relevant calculations take into account exponentially decaying contribution of the spin-dependent scattering from the interfaces. This modeling applies to one orientation of the electron spin. It shows a strong effect of the scattering from the interfaces on the effective conductivity.

\section{CONCLUSIONS}

We proposed a new magnetically controlled photodetector, which is based on a semiconductor $p-i-n$ structure covered on both sides with ferromagnetic metallic films. The proposed photodetector can be much more sensitive to the magnetic field than the magnetoresistive counterpart, even though they explore the same effect of GMR. The enhancement in the magnetic sensitivity is attributed to the generated spin-polarized carriers which have transport properties more sensitive to the spin-dependent scattering from defects at the interfaces.

\section{ACKNOWLEDGEMENTS}

The work is partially supported by the NATO Science fellowship CP(UN)06/B/2001/PO, and by the Polish State Committee for Scientific Research through the Research Project 5 P03B 09120.

\section{REFERENCES}

1. S. A. Wolf, D. D. Awschalom, R. A. Buhrman, J. M. Daughton, S. von Molnar, M. L. Roukes, A. Y. Chtchelkanova, and D. M. Treger, Science 294, 1488 (2001).

2. G. Schmidt, G. Richter, P. Grabs, C. Gould, D. Ferrand, and L. W. Molenkamp, Phys. Rev. Lett. 87, 227203 (2001).

3. H. Ohno and F. Matsukura, Sol. State Communs 117, 179 (2001).

4. E. I. Rashba, Phys. Rev. B 62, R16267 (2000).

5. A. Fert and H. Jaffres. Phys. Rev. B 64, 184420 (2001).

6. P. M. Levy, In: Solid State Physics, edited by F. Seitz, D. Turnbull, H. Ehrenreich, (Academic, New York, 1994), vol. 47, p.367.

7. M. A. M. Gijs and G. E. W. Bauer, Adv. Phys. 46, 285 (1997).

8. J.-Ph. Ansermet, J. Phys. Cond. Matter. 10, 6027 (1998).

9. A. Barthelemy, A. Fert, and F. Petroff. In: Handbook of Magnetic Materials, edited by K. H. J. Buschow (Elsevier, Amsterdam, 1999), vol. 12.

10. I. Žutić, J. Fabian, and S. Das Sarma, Phys. Rev. B 64, 121201 (2001).

11. S. M. Sze. Physics of Semiconductor Devices (Wiley, New York, 1981). 